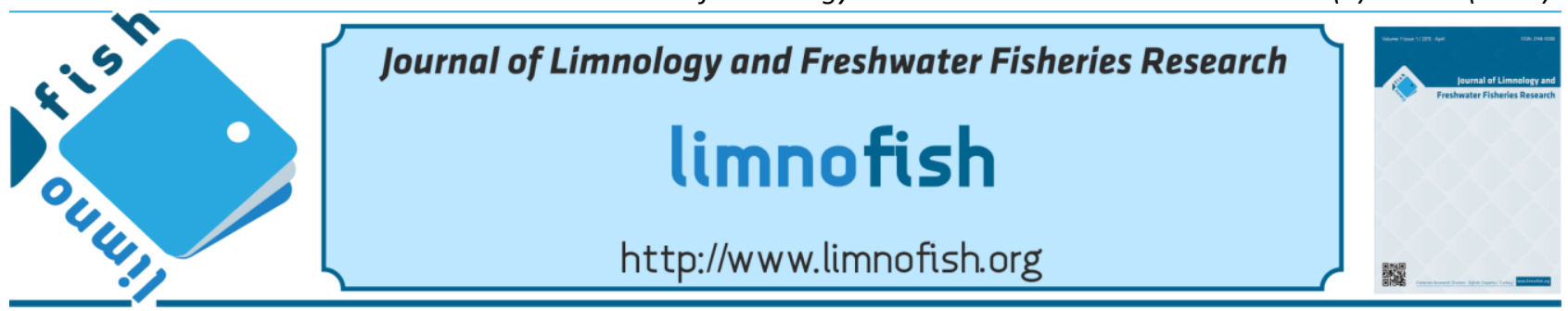

\title{
New Records for Distribution of Invasive Topmouth gudgeon (Pseudorasbora parva Temminck \& Schlegel, 1846) in Anatolia
}

\author{
Vedat YEĞEN ${ }^{1} *$, Rahmi UYSAL ${ }^{1}$, Abdulkadir YAĞCI ${ }^{1}$, Mehmet CESUR ${ }^{1}$, Soner ÇETINKAYA ${ }^{1}$, \\ Fuat BILGİN ${ }^{1}$, Hasan BOSTAN², Meral (Apaydın) YAĞCI ${ }^{1}$ \\ ${ }^{1}$ Fisheries Research Station, 32500, Eğirdir, Isparta-Türkiye \\ ${ }^{2}$ Ministry of Food, Agriculture and Livestock, County Directorate, 33640, Anamur, Mersin-Türkiye
}

\section{A B STR ACT}

The topmouth gudgeon Pseudorasbora parva is described as most invasive fish in Europe. It's a small cyprinid fish native to East Asia. In 1982, the existence of this species was first recorded from the Thrace region of Türkiye. Todays it is recorded from Thrace to Ceyhan region. In this study was carried out Afyonkarahisar, Bilecik, Burdur, Denizli, Eskişehir, Isparta, Konya, Kütahya and Uşak provinces between 2001 and 2013. Specimens collected from various localities in these provinces. Our results are shown $P$. parva has invaded many freshwater systems in Türkiye. This species has a potential threat to the diversity of the ichthyofauna of Anatolia and fish farms because of causing an increased mortality pressure on some salmonids and cyprinids, and totally inhibiting spawning of existing native fish.

Keywords: Freshwater fishes, invasive species, Pseudorasbora parva, Anatolia

\section{ARTICLE INFO}

$\begin{array}{ll}\text { SHORT COMMUNICATION } \\ \text { Received } & : 04.02 .2015 \\ \text { Revised } & : 18.03 .2015 \\ \text { Accepted } & : 19.03 .2015 \\ \text { Published } & : 20.04 .2015\end{array}$

\section{* CORRESPONDING AUTHOR}

vyegen@gmail.com

Tel : +902463133460

Fax: +90 2463133463

İstilacı Bir Tür Olan Çakıl Balığı (Pseudorasbora parva Temminck \& Schlegel, 1846)'nın Anadolu'daki Yayılımına Yeni Eklentiler

Öz: Çakıl Balığı Pseudorasbora parva Avrupa'nın en istilacı balık türü olarak tanımlanmaktadır. Doğu Asya kökenli, küçük cüsseli bir balıktır. Ülkemizdeki varlığı ilk defa 1982 yılında Trakya bölgesinden bildirilmiştir. Günümüzde Trakya'dan Ceyhan havzasına kadar geniş bir bölgede yayılım göstermektedir. Çalışmamız 2001 - 2013 yılları arasında Afyonkarahisar, Bilecik, Burdur, Denizli, Eskişehir, Isparta, Konya, Kütahya ve Uşak illerinde yürütülmüştür. Örnekler bu illerdeki çeşitli kaynaklardan elde edilmiştir. Çalışmamızın sonuçları $P$. parva istilasının ülkemizin tüm içsularını kaplamaya başladığını göstermektedir. Bazı Salmonid ve Cyprinid türlerinde ölümlerin artmasına ve doğal faunadaki türlerin üremelerini kısıtlamalarından dolayı Anadolu'nun ihtiyofaunası ve balık çiftlikleri açısından tehlike oluşturma potansiyeli bulunmaktadır

Anahtar kelimeler: Tatlısu balıkları, istilacı tür, Pseudorasbora parva, Anadolu

\section{Introduction}

The topmouth gudgeon Pseudorasbora parva (Temminck \& Schlegel, 1846) is a small cyprinid, originating from Japan, China, Korea and the River Amur catchment (Gozlan et al. 2002; Cakic et al. 2004; Witkovski 2011). It was first recorded for Europe in 1960, its inadvertent introduction mixed with herbivorous fishes (Ctenopharyngodon idella, Aristichthys nobilis, Hypophathalmichthys molitrix) imported from China to fish farms in neighbouring the lower course of the River Danube in 1961 and it has become distributed along this basin to other European countries (Erk'akan 1984; Caiola and Sostoa 2002; Gozlan et al. 2002; Pollux and Korosi 2006;
Witkovski, 2011). This fish species was first recorded from Thrace region in 1982, afterwards from Aksu stream in Anatolia in 1996 (Erk'akan 1984; Wildekamp et al. 1997). Today, it has invaded many freshwater environments from Thrace to Ceyhan basins (Balık et al. 2003; Şaşı and Balık 2003; Barlas and Dirican 2004; Ekmekçi and Kırankaya 2006; Uğurlu and Polat 2007; Ekmekçi and Kırankaya 2008; İlhan and Balık 2008; Yardım and Erdem, 2010; Apaydın Yağc1 et al. 2011; Meke et al. 2012; Çınar et al. 2013; Ekmekçi et al. 2013; İlhan and Sarı 2013; Özuluğ et al. 2013; Özeren and Arslan 2014). This study aimed to provide information about new localities by $P$. parva in Türkiye. 


\section{Materials and Methods}

Study area covers of Afyonkarahisar, Bilecik, Burdur, Denizli, Eskişehir, Isparta, Konya, Kütahya and Uşak provinces (Figure 1). Sampling was carried out at 507 stations included lakes, dams, ponds and streams by using different sampling equipment such as (gill nets, seine net, electroshocker) between 2001-2013 years. Samples were preserved in $4 \%$ formalin and collected in Eğirdir Fisheries Research Station.

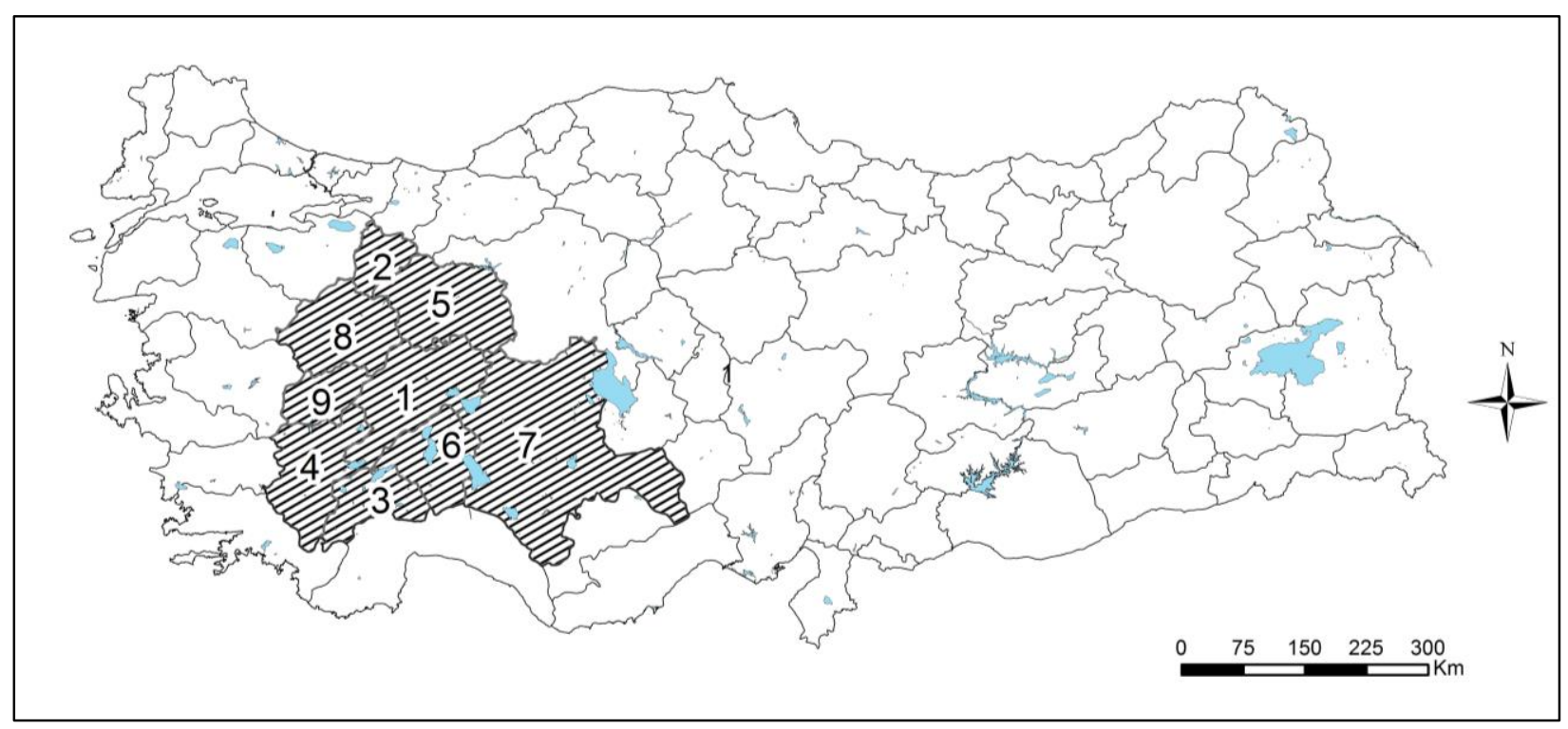

Figure 1. Study area (1.Afyonkarahisar, 2.Bilecik, 3.Burdur, 4.Denizli, 5.Eskişehir, 6.Isparta, 7.Konya, 8.Kütahya, 9.Uşak).

\section{Results}

We have obtained $P$. parva samples from 1 lake, 12 dams, 12 ponds and 2 stream in the Bilecik,
Burdur, Eskişehir, Isparta, Kütahya and Uşak provinces, any samples couldn't be obtained from Afyonkarahisar, Denizli, and Konya provinces (Table 1).

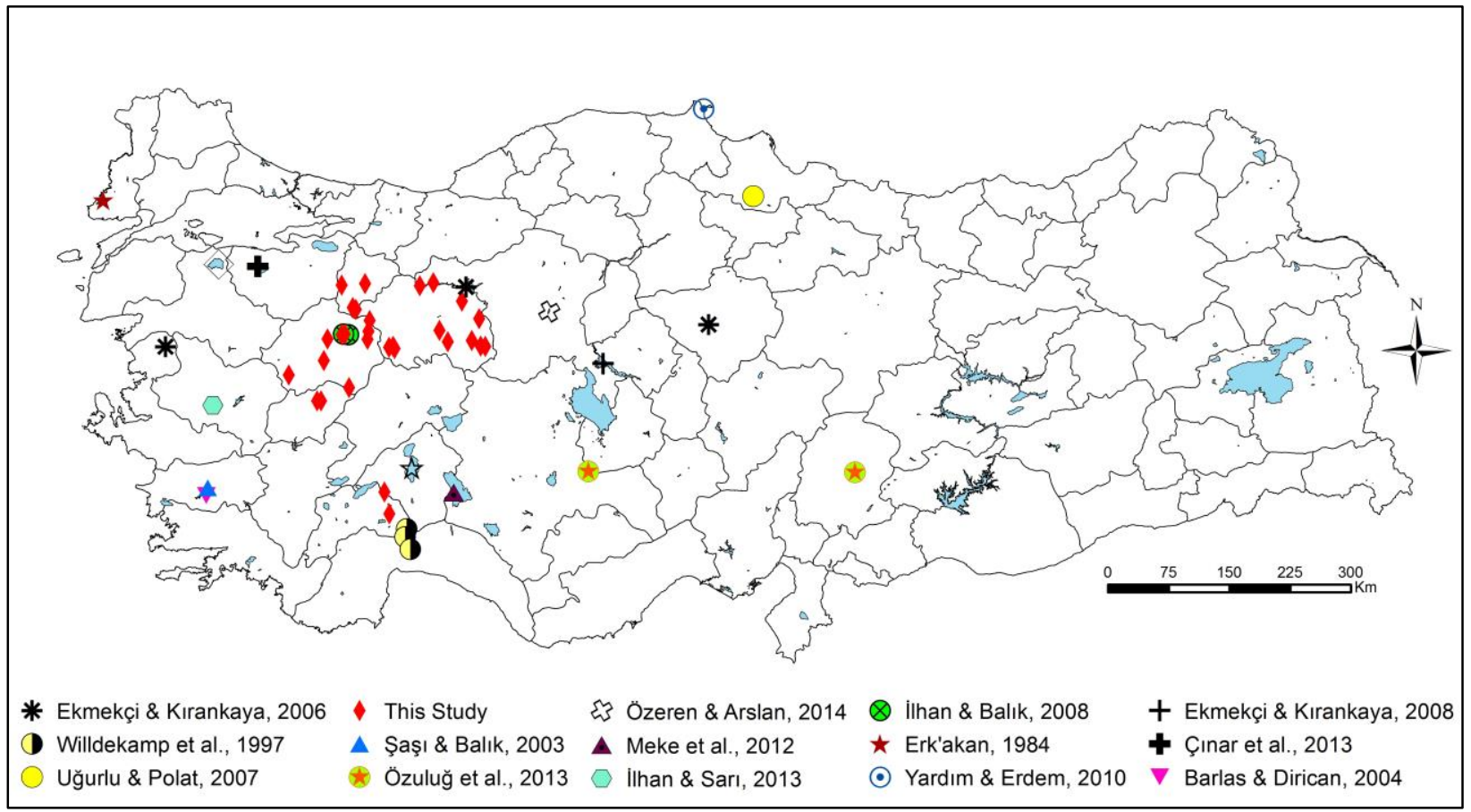

Figure 2. Distribution of P. parva in Türkiye. 
Table 1. Distribution of $P$. parva according to provinces.

\begin{tabular}{|c|c|c|c|}
\hline Province & Locality & Coordinates & Date \\
\hline \multirow{3}{*}{ Bilecik } & Dodurga Dam & $39^{\circ} 46^{\prime} 33^{\prime \prime} \mathrm{N}-2^{\circ} 57^{\prime} 36^{\prime \prime} \mathrm{E}$ & 18.08 .2010 \\
\hline & Kizıldamlar Dam & $40^{\circ} 02^{\prime} 24^{\prime \prime} \mathrm{N}-30^{\circ} 07^{\prime} 55^{\prime \prime} \mathrm{E}$ & 06.07 .2010 \\
\hline & Küçükelmalı Pond & $40^{\circ} 01^{\prime} 04^{\prime \prime} \mathrm{N}-29^{\circ} 47^{\prime} 28^{\prime \prime} \mathrm{E}$ & 06.05 .2010 \\
\hline Burdur & Onaç Dam & $37^{\circ} 29^{\prime} 58^{\prime \prime} \mathrm{N}-30^{\circ} 34^{\prime} 12^{\prime \prime} \mathrm{E}$ & 02.10 .2013 \\
\hline \multirow{12}{*}{ Eskişehir } & Çatören Dam & $39^{\circ} 19^{\prime} 25^{\prime \prime} \mathrm{N}-30^{\circ} 34^{\prime} 48^{\prime \prime} \mathrm{E}$ & 24.10 .2008 \\
\hline & Gökçekaya Dam & $40^{\circ} 01^{\prime} 57^{\prime \prime} \mathrm{N}-31^{\circ} 00^{\prime} 54^{\prime \prime} \mathrm{E}$ & 27.05.2009 \\
\hline & Kaymaz Dam & $39^{\circ} 32^{\prime} 32^{\prime \prime} \mathrm{N}-31^{\circ} 13^{\prime} 04^{\prime \prime} \mathrm{E}$ & 30.04 .2009 \\
\hline & Kunduzlar Dam & $39^{\circ} 21^{\prime} 22^{\prime \prime} \mathrm{N}-30^{\circ} 34^{\prime} 04^{\prime \prime} \mathrm{E}$ & 24.10 .2008 \\
\hline & Yenice Dam & $40^{\circ} 02^{\prime} 07^{\prime \prime} \mathrm{N}-30^{\circ} 55^{\prime} 48^{\prime \prime} \mathrm{E}$ & 26.05 .2009 \\
\hline & Bahçecik Pond & $39^{\circ} 25^{\prime} 16^{\prime \prime} \mathrm{N}-31^{\circ} 20^{\prime} 20^{\prime \prime} \mathrm{E}$ & 29.04 .2009 \\
\hline & Erenköy Pond & $39^{\circ} 45^{\prime} 09^{\prime \prime} \mathrm{N}-30^{\circ} 00^{\prime} 19^{\prime \prime} \mathrm{E}$ & 01.05 .2008 \\
\hline & Günyüzü II Pond & $39^{\circ} 22^{\prime} 28^{\prime \prime} \mathrm{N}-31^{\circ} 48^{\prime} 55^{\prime \prime E}$ & 28.04 .2009 \\
\hline & Koçaş II Pond & $39^{\circ} 26^{\prime} 20^{\prime \prime} \mathrm{N}-31^{\circ} 41^{\prime} 16^{\prime \prime} \mathrm{E}$ & 28.04 .2009 \\
\hline & Mercan Pond & $39^{\circ} 22^{\prime} 31^{\prime \prime} \mathrm{N}-31^{\circ} 52^{\prime} 54^{\prime \prime} \mathrm{E}$ & 29.04 .2009 \\
\hline & Akın Creek & $39^{\circ} 20^{\prime} 14^{\prime \prime} \mathrm{N}-30^{\circ} 30^{\prime} 15^{\prime \prime} \mathrm{E}$ & 13.09.2011 \\
\hline & Porsuk River & $39^{\circ} 40^{\prime} 38^{\prime \prime} \mathrm{N}-31^{\circ} 47^{\prime} 26^{\prime \prime E}$ & 16.08 .2011 \\
\hline Isparta & Gölcük Lake & $37^{\circ} 43^{\prime} 96^{\prime \prime} \mathrm{N}-30^{\circ} 29^{\prime} 94^{\prime \prime} \mathrm{E}$ & 17.05 .2003 \\
\hline \multirow{8}{*}{ Kütahya } & Çavdarhisar Dam & $39^{\circ} 10^{\prime} 29^{\prime \prime} \mathrm{N}-29^{\circ} 35^{\prime} 04^{\prime \prime E}$ & 19.08.2010 \\
\hline & Enne Dam & $39^{\circ} 28^{\prime} 29^{\prime \prime} \mathrm{N}-2^{\circ} 51^{\prime} 40^{\prime \prime} \mathrm{E}$ & 28.08 .2007 \\
\hline & Kayaboğazı Dam & $39^{\circ} 23^{\prime} 41^{\prime \prime N}-29^{\circ} 36^{\prime} 27^{\prime \prime E}$ & 30.08 .2007 \\
\hline & Söğüt Dam & $39^{\circ} 25^{\prime} 20^{\prime \prime} \mathrm{N}-30^{\circ} 11^{\prime} 25^{\prime \prime E}$ & 24.05 .2007 \\
\hline & Doğluşah Pond & $39^{\circ} 30^{\prime} 51^{\prime \prime} \mathrm{N}-30^{\circ} 12^{\prime} 05^{\prime \prime} \mathrm{E}$ & 24.05 .2007 \\
\hline & Dumlupınar Pond & $38^{\circ} 52^{\prime} 47^{\prime \prime} \mathrm{N}-29^{\circ} 57^{\prime} 00^{\prime \prime E}$ & 19.08.2010 \\
\hline & Pazarlar Pond & $38^{\circ} 59^{\prime} 38^{\prime \prime} \mathrm{N}-2^{\circ} 04^{\prime} 59^{\prime \prime} \mathrm{E}$ & 22.05 .2007 \\
\hline & Sekiören Pond & $39^{\circ} 27^{\prime} 52^{\prime \prime} \mathrm{N}-29^{\circ} 49^{\prime} 13^{\prime \prime E}$ & 03.05 .2007 \\
\hline \multirow{2}{*}{ Uşak } & Altıntaş Pond & $38^{\circ} 43^{\prime} 19^{\prime \prime} \mathrm{N}-29^{\circ} 30^{\prime} 01^{\prime \prime E}$ & 14.06 .2007 \\
\hline & Göğem Pond & $38^{\circ} 43^{\prime} 14^{\prime \prime} \mathrm{N}-29^{\circ} 33^{\prime} 23^{\prime \prime} \mathrm{E}$ & 14.06.2007 \\
\hline
\end{tabular}

\section{Discussion}

$P$. parva has expanded its range and has established successful populations in Turkish inland waters since 1982 (Figure 2). A discussion is how it passed the Bosphorus and Dardanelles. Its success can be explained by its life-history characteristics such as high reproductive effort and nest guarding behaviour, early sexual maturity (after 1 year), plasticity in spawning area preference, wide ecological tolerance, lack of enemy in its spreading areas and lower predation pressure due to its prefer to dense vegetated areas in littoral zone (Rosecchi et al. 2001; Gozlan et al. 2002; Witkowski 2011).

$P$. parva have negative impacts on the native fish fauna via competition spawning area, food and other sources (Ekmekçi et al. 2013). Additionally it is vector an deadly pathogen rosette agent Sphaerothecum destruens, which is responsible for increased mortality and inhibited spawning of some cyprinids and salmonids (Arkush et al. 1998; Gozlan et al. 2005; Pinder et al. 2005; Andreou et al. 2011, 2012). This pathogen were determined from Sarıçay Creek, Muğla in Türkiye (Ercan et al. 2013). Therefore P. parva is considered as a serious threat to both native and farmed fishes in Türkiye.

Recently control activities is focused on eradication of $P$. parva from natural waters in some European countries, particularly in England (Allen et al. 2006; Britton and Brazier 2006; Britton et al. 2008, 2009, 2010). Also there are numerous researches about biological properties and genetic structure of $P$. parva in Türkiye (Ăgdamar et al. 2013; Akbaş et al. 2013; Kırankaya et al. 2013; Özdilek et al. 2013). Anatolia is accepted as a continental land in terms of biodiversity. Initiation of an effective fight against this species is highly crucial for both freshwater fish fauna and aquaculture sector in our country. In other 
words, effective reduction strategies must be created and put into practice utilizing the information obtained from studies on the species.

\section{Acknowledgements}

This study was supported by General Directorate of Agricultural Research and Policies with TAGEM/HAYSÜD/2001/09/02/01 and TAGEM/HAYSÜD/2006/06/05/01 projects and was represented in $6^{\text {th }}$ Limnology Symposium, 25-28 August 2014, Bursa-Türkiye by oral presentation.

\section{References}

Ağdamar S, Tarkan AS, Keskin E. 2013. İstilacı bir türün yüksek adaptasyon yeteneğinin altında yatan neden: Genetik çeşitlilik ve Pseudorasbora parva örneği. Paper presented at: Türkiye İstilacı Tatlısu Türleri Çalıştayı: Ulusal Eylem Planı; İstanbul, Türkiye. [in Turkish]

Akbaş F, Tarkan, AS, Top N, Karakuş U, Gaygusuz Ö, Dorak Z, Aydın H. 2013. Yabanc1 bir tür olan Pseudorasbora parva'nın büyüme ve hayat döngüsü özelliklerinin farklı ekosistemlerde karşılaştırmalı olarak incelenmesi. Paper presented at: Türkiye İstilacı Tatlısu Türleri Çalıştayı: Ulusal Eylem Planı; İstanbul, Türkiye [in Turkish]

Allen Y, Kirby S, Copp GH, Brazier M. 2006. Toxicity of rotenone to topmouth gudgeon Pseudorasbora parva for eradication of this non-native species from a tarn in Cumbria, England. Fisheries Manag Ecol. 13(5):337-340. doi: 10.1111/j.1365-2400.2006.00499.x

Andreou D, Arkush KD, Guegan, JF, Gozlan RE. 2012. Introduced pathogens and native freshwater biodiversity: A Case Study of Sphaerothecum destruens. PLoS ONE. 7(5):e36998. doi: 10.1371/journal.pone.0036998

Andreou D, Gozlan RE, Stone D, Martin P, Bateman K, Feist SW. 2011. Sphaerothecum destruens pathology in Cyprinids. Dis Aquat Organ. 95(2):145-151. doi: 10.3354/dao02356

Apaydın Yağcı M, Bostan H, Yağcı A, Yeğen V, Uysal R, Bilgin F. 2011. Hizla yayılmaya devam eden istilacı bir tür örneği: Eğirdir Gölü'nden (Pseudorasbora parva Temminck \& Schlegel, 1846). Paper presented at: 16. Su Ürünleri Sempozyumu; Antalya, Türkiye. [in Turkish]

Arkush KD, Frasca S, Hedrick RP. 1998. Pathology associated with the rosette agent, a systemic protist infecting salmonid fishes. J Aquat Anim Health. 10:1-11. doi: 10.1577/15488667(1998)010<0001:PAWTRA>2.0.CO;2

Balık S, Sarı HM, İlhan A, Topkara ET. 2003. Susurluk ırmağı havzası balık faunasının araştırılması. Ege University Fisheries Faculty Basic Sciences Department Project No: 2003/SÜF/003 [in Turkish]

Barlas M, Dirican S. 2004. The fish fauna of the DipsizÇine (Muğla-Aydın) stream. Gazi Uni J Sci. 17(3):35-48.
Britton JR, Brazier M. 2006. Eradicating the invasive topmouth gudgeon, Pseudorasbora parva, from a recreational fishery in Northern England. Fisheries Manag Ecol. 13(5):329-335. doi: 10.1111/j.1365-2400.2006.00510.x

Britton JR, Davies GD, Brazier M. 2008. Contrasting life history traits of invasive Topmouth gudgeon (Pseudorasbora parva) in adjacent ponds in England. J Appl Ichthyo. 24(6):694-698. doi: 10.1111/j.1439-0426.2008.01163.x

Britton JR, Davies GD, Brazier M. 2009. Eradication of the invasive Pseudorasbora parva results in increased growth and production of native fishes. Ecol Freshw Fish. 18(1):8-14. doi: 10.1111/j.1600-0633.2008.00334.x

Britton JR, Davies GD, Brazier M. 2010. Towards the successful control of Pseudorasbora parva in the UK. Biol Invasions. 12(1):125-131. doi: 10.1007/s10530-009-9436-1

Caiola N, Sostoa AD. 2002. First record of the Asiatic Cyprinid Pseudorasbora parva in the Iberian Peninsula. J Fish Biol, 61(4):1058-1060. doi: 10.1006/jfbi.2002.2103

Cakic P, Lenthard M, Kolarevic J, Mikovic B, Hegedis A. 2004. Distribution of the Asiatic Cyprinid Pseudorasbora parva in Serbia and Montenegro. J Fish Biol. 65:1431-1434. doi: 10.1111/j.1095-8649.2004.00525.x

Çınar Ş, Küçükkara R, Balık İ, Çubuk H, Ceylan M, Erol KG, Yeğen V, Bulut C. 2013. Uluabat (Apolyont) Gölü'ndeki balık faunasının tespiti, tür kompozisyonu ve ticari avcılığın türlere göre dağılımı. J Fish Sci. 7(4):309-316. doi: 10.3153/jfscom.2013034 [In Turkish]

Ekmekçi FG, Kırankaya ŞG. 2006. Distribution of an invasive fish species, Pseudorasbora parva (Temminck \& Schlegel, 1846) in Turkey. Turk J Zool. 30(3):329-334.

Ekmekçi FG, Kırankaya ŞG. 2008. Türkiye içsularındaki istilacı balık türlerinin biyolojik çeşitlilik ile balıkçılığa etkileri ve ulusal eylem planının oluşturulması. Paper presented at: III. Limnology Symposium; İzmir, Türkiye. [in Turkish]

Ekmekçi FG, Kırankaya, ŞG, Gençoğlu L, Yoğurtçuoğlu B. 2013. Türkiye içsularındaki istilacı balıkların güncel durumu ve istilanın etkilerinin değerlendirilmesi. İstanbul Uni Su Ürünleri Derg. 28(1):105-140 [in Turkish]

Ercan MD, Öntaş C, Baba E, Karakuş U, Top N, Tarkan AS. 2013. İstilacı bir tatlısu balığı türünün (pseudorasbora parva) Türkiye içsularında balıkçılık ve biyoçeşitliliği tehdit eden bir paraziti taşıyıcılığı. Paper presented at: Türkiye İstilacı Tatlısu Türleri Çalıştayı, Ulusal Eylem Planı; İstanbul, Türkiye [in Turkish]

Erk'akan F. 1984. Trakya bölgesinden Türkiye için yeni kayıt olan bir balık türü Pseudorasbora parva (PiscesCyprinidae). Doğa Bilim Derg. A2:350-351. [in Turkish]

Gozlan RE, Pinder AC, Shelley J. 2002. Occurrence of the Asiatic Cyprinid Pseudorasbora parva in 
England. J Fish Biol. 61(1):298-300. doi: 10.1006/jfbi.2002.2042

Gozlan RE, St-Hilaire S, Feist SW, Martin P, Kent ML. 2005. Biodiversity: Disease threat to European fish. Nature. 435(7045):1046. doi: 10.1038/4351046a

İlhan A, Balık S. 2008. Fish fauna of the inland waters in Western Black Sea Region. Ege Uni J Fish Aqua Sci. 25(1):75-82.

İlhan A, Sarı HM. 2013. İstilacının kıskacında; Marmara Gölü. Paper presented at: Türkiye İstilacı Tatlısu Türleri Çalıştayı, Ulusal Eylem Planı; İstanbul, Türkiye [in Turkish]

Kırankaya ŞG, Ekmekçi FG, Özdilek (Yalçın) Ş, Yoğurtçuoğlu B, Gençoğlu L. 2013. Hirfanlı Baraj Gölü'nde bulunan istilacı balık türü Pseudorasbora parva (Temminck\&Schlegel, 1846)'nın yaşam döngüsü özelliklerinin incelenmesi. Paper presented at: Türkiye İstilacı Tatlısu Türleri Çalıştayı, Ulusal Eylem Planı; İstanbul, Türkiye [in Turkish]

Meke T, Küçükkara R, Çınar Ş, Çavdar N, Babar B, Yener O. 2012. Beyşehir Gölü balık faunası için yeni bir kayıt: istilac1 bir tür: Pseudorasbora parva (Temminck \& Schlegel, 1846). Paper presented at: V. Limnology Symposium; Isparta, Türkiye. [in Turkish]

Özdilek (Yalçın) Ş, Kırankaya ŞG, Ekmekçi FG. 2013. Feeding ecology of the Topmouth gudgeon Pseudorasbora parva (Temminck and Schlegel, $1846)$ in the Gelingüllü reservoir, Turkey. Turk J Fish Aquat Sc. 13(1):87-94. doi: 10.4194/1303-2712-v13_1_11

Özeren SC, Arslan P. 2014. Mogan Gölü'nde yaşayan Pseudorasbora parva'nın Ligula intestinalis ile enfekte olmuş ve olmamış bireylerinde kondisyon faktörü karşılaştırılması. Paper presented at: 22. National Biology Congress; Eskişehir, Türkiye. [in Turkish]
Özuluğ M, Saç G, Gaygusuz Ö. 2013. New distribution areas for invasive Gambusia holbrooki, Carassius gibelio ve Pseudorasbora parva (Teleostei) from Turkey. Istanbul Uni J Fish Aqua Sci. 28(1): 1-22. [in Turkish]

Pinder AC, Gozlan RE, Britton JR. 2005. Dispersal of the invasive topmouth gudgeon, Pseudorasbora parva in the UK: a vector for an emergent infectious disease. Fisheries Manag Ecol. 12(6):411-414. doi: 10.1111/j.1365-2400.2005.00466.x

Pollux BJA, Korosi A. 2006. On the occurrence of the Asiatic Cyprinid Pseudorasbora parva in the Netherlands. J Fish Biol. 69(5): 1575-1580. doi: 10.1111/j.1095-8649.2006.01218.x

Rosecchi E, Thomas F, Crivelli AJ. 2001. Can life history traits predict the fate of introduced species? A case study on two Cyprinid fish in Southern France. Freshwater Biol. 46(6):845-853. doi: 10.1046/j.1365-2427.2001.00715.x

Şaşı H, Balık S. 2003. The distribution of three exotic fishes in Anatolia. Turk J Zool. 27(4):319-322.

Uğurlu S, Polat N. 2007. Exotic fish species inhabiting in freshwater sources within the province of Samsun. Journal of Fish Sci. 1(3):139-151. doi: 10.3153/jfscom.2007017 [in Turkish]

Wildekamp RH, Van Neer W, Küçük F, Ünlüsayın M. 1997. First record of the eastern Asiatic gobionid fish Pseudorasbora parva from the asiatic part of Turkey. J Fish Biol. 51(4):858-868. doi: 10.1111/j.1095-8649.1997.tb02006.x

Witkowski A. 2011. Nobanis - Invasive alien species fact sheet Pseudorasbora parva; [cited 2015 Jan 15]. Available from http://www.nobanis.org/

Yardım Ö, Erdem Y. 2010. Sinop ilinde bulunan bazı lentik sulardaki istilacı balık türlerinin durumu. Paper presented at: 4th National Limnology Symposium; Bolu, Türkiye. [in Turkish] 